\title{
COMPARATIVE ANALYSIS OF ENERGY DETECTION AND ARTIFICIAL NEURAL NETWORK FOR SPECTRUM SENSING IN COGNITIVE RADIO
}

\author{
Sanjog Shah and R.G. Yelalwar \\ Department of Electronics and Telecommunication Engineering, Pune Institute of Computer Technology, India
}

\begin{abstract}
In today's wireless communication technology, spectrum occupancy is one of the major challenge. To perform all the task in wireless communication intelligently, Cognitive Radio (CR) is used. With the help of machine learning techniques, performance of $C R$ will increase. In this paper, implementation of spectrum sensing (SS) in Cognitive Radio Network (CRN) is presented. To check the availability of spectrum, the supervised Machine Learning (ML) and conventional spectrum sensing method is used. To classify signal and noise, the Artificial Neural Network (ANN) classifier is used. The classifier's result shows better result than conventional method's result.
\end{abstract}

\section{Keywords:}

Machine Learning, Cognitive Radio Network, Cognitive Radio Network

\section{INTRODUCTION}

In today's modern generation, a magnificent spread, development of wireless communications as well as the exposure of new communication technology, protocol is experienced. As increasing the demand of spectrum, it is probable that availability of some spectrums will very few. Whereas some spectrums remain unused [1]. The national body, Federal Communications Commission (FCC) is coordinate, consumption of radio spectrum resources and the regulation of radio emissions [2]. To utilize spectrum efficiently, the Cognitive Radio (CR) concept was introduced.

The CR is intelligent radio whereas any radio can be intelligent by fulfilling three conditions. This conditions are perception, learning, and reasoning. CR fulfill all this conditions. $\mathrm{CR}$ is wireless communication device which can act as transceiver and can identify the spectrum availability status. In CR, the available spectrum is sensed and later analyzed to find if there is availability of spectrum holes. Assignment of available spectrum is done opportunistically on the basis of spectrum analysis to the Secondary User (SU). All the other operations of CR are highly dependent on precise sensing of available spectrum which makes $\mathrm{SS}$ as the most crucial part of CR [1].

The Cognitive Radio Network (CRN) consist of primary users (PU) and SU. A user who has legacy rights on the usage of a specific part of the spectrum is called PUs. PUs has higher priority to use spectrum. A user who doesn't has legacy rights on the usage of a specific part of the spectrum is called SUs. SUs has lowest priority to use spectrum [2]. Spectrum Sensing (SS) is basic requirement in CRN. It is challenging task in CR systems. Some conventional SS methods are explained in [1]. In this paper, energy detection method is explained. Other than conventional methods, SS can be done using ML methods. The accuracy of SS is better using ML methods than conventional methods.
Michell defined ML as, a computer program is said to learn from experience $E$ (past data) with respect to some class of task $T$ and performance measured $P$. Learning is the ability to improve behavior based on experience. The ML is mainly divided into two categories, supervised ML and unsupervised ML. Supervised ML has input with labeled data, labeled data contains desired output of respective input. Whereas unsupervised ML does not have any labeled data. In unsupervised ML the input data is divided into clusters. ML technique is useful for classification, prediction as well as decision making. In supervised machine learning technique, the input data is given to system with respect to its labeled data for training. The input data can be linear or nonlinear. Based on data, the one of the supervised learning technique will selected. After the training, system will classify the data. Then decision will take and data will classify in one of the available class. The performance of system depends upon, the algorithm which is selected and its parameters and their value. For example, SVM's major parameter is its kernel function and each kernel function will have set of values, in which they give different results. In artificial neural network (ANN), type of ANN and number of hidden layers are important. The survey of ML techniques is mentioned [4] and survey of ML for CRN are explained in [3].

\section{RELATED WORKS}

SS is effected by multi path and shadowing which shows degradation of system performance. The system gives different results over different fading channels like AWGN, Rayleigh, Nakagami [2]. By using SVM, ANN, k means techniques, system performance can be improved but there are different challenges, advantages and limitations in different techniques [3]. To overcome the challenges some modern ML are developed in CR. There are different types and variants of Artificial Intelligence (AI) and machine learning algorithms that can be used in CR such as fuzzy logic system (FLS) for decision making [4], neural network model for prediction of spectrum holes [5]-[8]. The stateof-the-art of machine-learning techniques in cognitive radios is presented in [8]. A novel Cooperative Spectrum Sensing (CSS) algorithms for CRN based on ML techniques which are used for pattern classification are explained in [11] and new approach of ANN based SS is proposed in [9].

\section{MAIN CONTRIBUTION}

In this paper, we have considered AM, FM and GSM 900 spectrum along with Amplitude Modulation (AM), Frequency Modulation (FM) and Binary Phase Shift Keying (BPSK) modulation for received signal respectively. By considering different sampling rate, the signals are represented with different 
number of samples. For signal classification robust dataset is created by considering four different features namely energy, autocorrelation, standard deviation (STD) and energy spectral density (ESD) features. Along with these features, signal samples and different SNR values are also considered for creation of robust dataset.

\section{PROPOSED SYSTEM}

In this paper, the system is implemented using ED and ANN by considering AM, FM and BPSK signal for $L=100,200$ and 300 samples. Every feature will have its own significance so as mentioned in section 3 , four features of received signal extracted. The energy of received signal is easy to compute and by comparing with the threshold value the signal can be easily classify. The autocorrelation is used to check the presence of a periodic signal obscured by noise. The most obscured by noise signals do not show a well-defined peak-to-peak value and have a random nature so STD describes how much the peak value of signal differs from its mean value. Energy spectral density describes how the energy of a signal is distributed with frequency. The features are calculated as follows shown in Eq.(1) to Eq.(4), which describes as the nth signal taken for the $i^{\text {th }}$ frequency band.

$$
\begin{gathered}
E_{i}(n)=\sum_{n=-\infty}^{\infty}\left|y_{i}(n)^{2}\right| \\
A_{i}(n)=\sum_{n=-\infty}^{\infty}\left|y_{i}(n) * y_{i}(n-k)\right| \\
T_{i}(n)=\sqrt{\frac{1}{n} \sum_{j=1}^{n}\left(\mu_{i j}-y_{i}(n)\right)^{2}} \\
\operatorname{ESD}_{i}(n)=\operatorname{FFT}\left(A_{i}(n)\right)
\end{gathered}
$$

where $\mu=$ mean of signal

\subsection{ENERGY DETECTION}

In SS, Energy Detection (ED) is one of the most common approach because of its low implementation and computational complexities. It is more generic as receivers do not need any knowledge on the primary user's signal. Some of the shortcoming which need to be overcome with energy detector based sensing are selection of the threshold for detecting primary users, Inability to differentiate interference from primary users and noise, and poor performance under low signal-to-noise ratio (SNR) values [1].

The Fig.1 shows Energy detection method, in which the received signal is transmitted through band pass filter (BPF) then energy of signal obtained by squaring the amplitude of signal and it is integrated over time and by comparing with threshold, the decision will take weather Primary User (PU) is present or not [2].

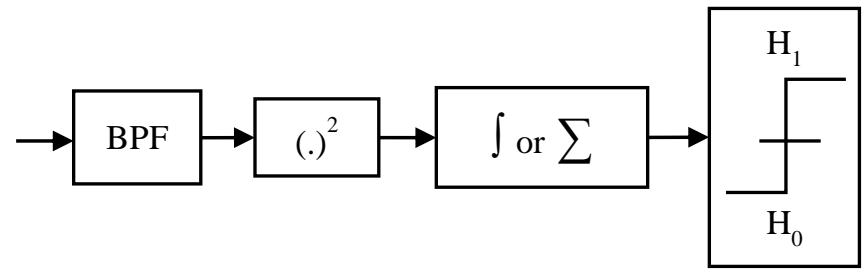

Fig 1. Energy Detection Method
In ED, $P_{f a}$ should be kept as small as possible in order to prevent underutilization of transmission opportunities. Usually, $P_{f a}$ kept input to the system and measure the $P_{d}$ of the system. The decision threshold, $\lambda$ can be selected for finding an optimum balance between Pd and Pfa. However, this requires knowledge of noise and detected signal powers. The noise power can be estimated but the signal power is difficult to estimate as it changes depending on on-going transmission characteristics and the distance between the cognitive radio and primary user. In practice, the threshold is chosen to obtain a certain false alarm rate. Hence knowledge of noise variance is sufficient for selection of a threshold.

The received signal is considered in binary hypothesis shown in Eq.(5) and Eq.(6).

$$
\begin{aligned}
& H_{0}: y(n)=N(n) \\
H_{1}: y(n)= & S(n)+N(n), P U \text { is present }
\end{aligned}
$$

where $S(n)=$ Transmitted Signal by $P U$.

$$
N(n)=\mathrm{AWGN}
$$

The main objective of spectrum sensing is to decide whether the received signal by secondary user (SU) is $H_{0}$ or $H_{1}$.

The energy of signal is calculated as shown in Eq.(7)

$$
E=\sum_{i=1}^{L}\left|y(n)^{2}\right|
$$

After energy value, a threshold value is calculated as shown in Eq.(8) for the comparison.

$$
\lambda=\frac{Q\left(P_{f a}\right)}{\sqrt{L}}
$$

By comparing $E$ and $\lambda$ value the decision of PU signal is taken. If $E \geq \lambda$ then PU is present otherwise PU is absent.

The performance of the system is measured on terms of the three probabilities: $P_{d}, P_{f a}$ and $P_{e} . P_{d}$ is the probability of detecting a PU signal when hypothesis $H_{1}$ is true. Thus, a large detection probability is desired. It is formulated as shown in Eq.(9),

$$
P_{d}=P\left(E>\lambda \mid H_{1}\right)
$$

The probability of miss is calculated as shown in Eq.(10)

$$
P_{\text {miss }}=1-P_{d}
$$

The Eq.(11) shows total error rate of system i.e. $P_{e}$ is calculated as:

$$
P_{e}=P_{f a}+P_{m i s s}
$$

Algorithm 1 states the core steps that are executed in Matlab for performance measurement. The symbols and notification that are used in the algorithm are explained below.

$L=$ Number of signal sample; $t=$ Total time period of signal; $f_{c}=$ Carrier frequency of signal; $f_{m}=$ Modulating frequency of signal; $s_{\text {mod }}=$ Bipolar NRZ signal; $m_{i}=$ Modulation Index; $x_{c}=$ PU signal (AM or FM or BPSK); $P_{f}=$ Probability of False Alarm; $k=$ Monte carlo simulation; $x_{c t}=$ Received signal; $E=$ Energy of signal; $i=$ Counter; $P_{d}=$ Probability of detection; $P_{m i s s}=$ Probability of miss detection; $P_{e}=$ Probability of error or error rate.

\section{Algorithm 1: ED algorithm}

Input: $L, t, f_{c}, f_{m}, s_{m o d}, m_{i}, x_{c}, P_{f}$

For $k$ times do 
consider received signal as follows

simulate noise $=a w g n$

$x_{c t}=x_{c}+$ noise

for all $L$

$$
E=E+\left(x_{c t}\right)^{2}
$$

end

thresh $=\operatorname{erfc} \frac{P_{f}}{\sqrt{2}}$

if thresh $\geq E$ then

$$
i=i+1
$$

end

$P_{d}=i / k$

$P_{\text {miss }}=1-P_{d}$

$P_{e}=P_{\text {miss }}+P_{f}$

end

\subsection{ARTIFICIAL NEURAL NETWORK}

ANN is a ML technique that inspired from human brain and consists of a number of artificial neurons. In ANN neuron will have less connection than biological neuron. Each neuron in ANN receives a number of inputs. To update the neuron, the activation function are used as unit step function, linear transfer function and sigmoid transfer function. The linear transfer function is used when the inputs are linearly separable and sigmoid transfer function is used when inputs are non-linearly separable.

The neuron is the basic information processing unit of a NN. It consists of a set of the neuron inputs $X_{1}, X_{2}, \ldots, X_{n}$, with weights $W_{1}, W_{2}, \ldots, W_{m}$ and an adder function (linear combiner) for computing the weighted sum of the inputs [10].

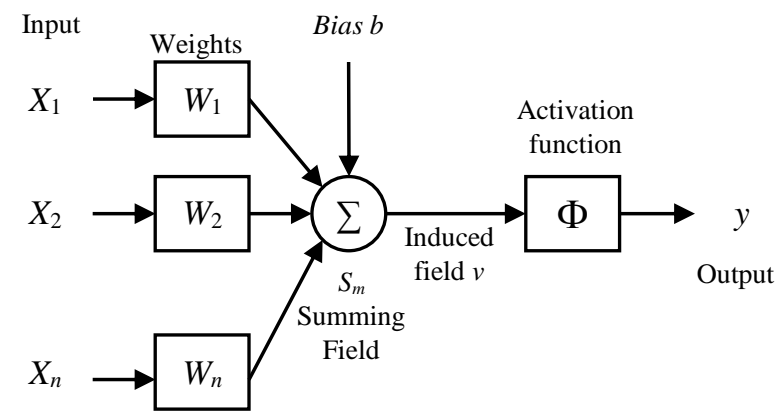

Fig.2. Neuron diagram

In ANN system, the Multilayer Feed Forward Neural Network (MFFNN) is used in for classification of PU signal and noise so binary hypothesis is considered as shown in Eq.(5) and Eq.(6).

The received signal is considered by $L=100,200$ and 300 number of samples. The Energy, STD, Autocorrelation and ESD these features of signal are extracted for classification and created input array as $\left\{x_{11}, x_{12}, \ldots, x_{k m}\right\}$, labeled data $\left\{y_{1}, y_{2}, \ldots, y_{k}\right\}$ for corresponding input. They are arranged in two matrices i.e. Input Matrix $(X)$ and Target Matrix $(T)$. These are defined as:

$$
\begin{gathered}
X=\left[\begin{array}{ccc}
x_{11} & \ldots & x_{1 m} \\
\vdots & \ddots & \vdots \\
x_{k 1} & \ldots & x_{k m}
\end{array}\right] \\
T=\left[\begin{array}{cc}
y_{1} & \bar{y}_{1} \\
\vdots & \vdots \\
y_{k} & \bar{y}_{k}
\end{array}\right]
\end{gathered}
$$

The matrix $X$ size is $\left(k^{*} m\right)$ where $k$ is a number of received signals and $\mathrm{m}$ is an index of the feature. The indexing is done as

$$
\begin{gathered}
m=1 \text { for Energy of } y(n) \\
m=2 \text { for STD of } y(n) \\
m=3 \text { for Autocorrelation of } y(n) \\
m=4 \text { for ESD of } y(n)
\end{gathered}
$$

As shown in Fig.2, neuron consists of a set of the input matrix $X$ with weights $W_{1}, W_{2}, \ldots, W_{n}$ and an adder function (linear combiner) for computing the weighted sum of the inputs. The $70 \%$ samples from $X$ are selected for training and $15 \%$ are used for validation.

$$
S_{m}=\sum_{j=0}^{m} W_{j} x_{j}
$$

The Eq.(12) shows weighted sum $S_{m}$

Eq.(13) shows the bias $b$ has the effect of applying a transformation to the weighted sum $S_{m}$.

$$
v=S_{m}+b
$$

where $v$ is called the induced field of the neuron.

The sigmoid transfer function is applied on Eq.(13) and we get output y of system as shown in Eq.(14)

$$
\Phi(v)=\log \operatorname{sig}\left(S_{m}+b\right)=g
$$

As mention above, the MFFNN is used, so compare $g$ with Target matrix $T$, if $g$ is equivalent to $T$ then do not update weight and give it to next layer otherwise update weight and give it to next layer. The weight can be updated as shown in Eq.(15) and Eq.(16).

$$
\begin{aligned}
\Delta w_{i} & =\left(y_{i}-\bar{y}_{i}\right) * \\
W_{i} & =W_{i}+\Delta w_{i}
\end{aligned}
$$

The remaining $15 \%$ features are used for testing; these features have no effect on training. So it provides an independent measure of network performance. $P_{d}$ and $P_{f a}$ is calculated as shown in Eq.(17) and Eq.(18) respectively

$$
\begin{gathered}
P_{d}=\frac{y \in\{1\} \text { when } T \in\{1\}}{T \in\{1\}} \\
P_{f a}=\frac{y \in\{1\} \text { when } T \in\{-1\}}{T \in\{-1\}}
\end{gathered}
$$

The above system is implemented same as for AM and FM signal also and similarly for only one energy feature (single feature).

Algorithm 2 states the core steps that are executed in Matlab for performance measurement. The symbols and notification that are used in the algorithm are explained below. $S N R=$ Signal to Noise Ratio in linear; $L=$ Number of signal sample; $t=$ Total time 
period of signal; $f_{c}=$ Carrier frequency of signal; $f_{m}=$ Modulating frequency of signal; $s_{\text {mod }}=$ Bipolar NRZ signal; $m_{i}=$ Modulation Index; $x_{c}=$ PU signal (AM or FM or BPSK); $j=$ Counter for number simulations; $k=$ Number of Signals; $x_{c t}=$ Received signal; $S_{E}=$ Energy of Signal; SSTD = Standard Deviation of Signal; $S_{\text {Autocorr }}=$ Autocorrelation of Signal; SESD $=$ Energy Spectral Density of Signal; $N E=$ Energy of Noise; $N S T D=$ Standard Deviation of Noise; $N_{\text {Autocorr }}=$ Autocorrelation of Noise; $N E S D=$ Energy Spectral Density of Noise; $y_{i}=$ Target values (Labeled data); $i=$ Number of features; $P_{f}=$ Probability of False Alarm; $P_{d}$ $=$ Probability of detection; $P_{\text {miss }}=$ Probability of miss detection; $P_{e}=$ Probability of error or error rate.

\section{Algorithm 2: ANN algorithm}

Input: $S N R, L, t, f_{c}, f_{m}, s_{m o d}, m_{i}, x_{c}$

for $j$ times do

for $k$ times do

consider $f_{m}(k)$ or $s_{\text {mod }}(k)$

simulate noise $=$ awgn

$x_{c t}(k)=x_{c}+$ noise

end

for $k$ time do

$$
\begin{aligned}
& S E=\left|x_{c t}(k)\right|^{2} \\
& S S T D=\operatorname{std}\left(x_{c t}(k)\right) \\
& S_{\text {Autocorr }}=\operatorname{auto}\left(x_{c t}(k)\right) \\
& S E S D=\operatorname{esd}\left(x_{c t}(k)\right)
\end{aligned}
$$

end

for $k$ time do

$$
\begin{aligned}
& N E=\mid \text { noise }\left.(k)\right|^{2} \\
& N S T D=\operatorname{std}(\operatorname{noise}(k)) \\
& N_{\text {Autocorr }}=\operatorname{auto}(\operatorname{noise}(k)) \\
& N E S D=\operatorname{esd}(\operatorname{noise}(k))
\end{aligned}
$$

end

signal_features $=\left[S E, S S T D, S_{\text {Autocorr }}, S E S D\right]$

noise_features $=\left[N E, N S T D, N_{\text {Autocorr }}, N E S D\right]$

class $_{1}=$ create $2 k$ times 1

$\operatorname{class}_{1}=\operatorname{class}_{1}(k+1: 2 k)=-1$

class $_{2}=$ create $2 k$ times -1

$\operatorname{class}_{2}=\operatorname{class}_{2}(\mathrm{k}+1: 2 k)=1$

class $=\left[\right.$ class $_{1}$, class $\left._{2}\right]$

Apply ANN

for $i$ times do

$$
\begin{aligned}
& g_{i}=\log \operatorname{sig}\left(S_{m}+b\right) \\
& \Delta w_{i}=\left(g_{i}-\bar{y}_{i}\right) * x_{i} \\
& W_{i}=W_{i}+\Delta w_{i} \\
& \text { Error }=y_{i} \text {-class }(i)
\end{aligned}
$$

end

misclassification $=1-\operatorname{sum}($ Error $=0)$

$P_{e}=$ misclassification $/ 1$

$$
\begin{aligned}
& P_{d}=\frac{y \in\{1\} \text { when } T \in\{1\}}{T \in\{1\}} \\
& P_{f a}=\frac{y \in\{1\} \text { when } T \in\{-1\}}{T \in\{-1\}}
\end{aligned}
$$

Pmiss $=1-\mathrm{Pd}$

end

\section{SIMULATION RESULTS AND DISCUSSION}

In this system, the SU will receive the signal from different frequency bands which are AM, FM and GSM900. In the AM band, the AM modulation is considered with $f_{m}=3 \mathrm{kHz}$ to $5 \mathrm{kHz}$, $f_{c}=540 \mathrm{kHz}$, for FM band, FM modulation is considered with $f_{m}$ $=5 \mathrm{kHz}$ to $15 \mathrm{kHz}, f_{c}=100 \mathrm{kHz}$ and in GSM900 band, BPSK modulation is considered with $s_{\text {mod }}=10$ bits and $f_{c}=900 \mathrm{MHz}$. The $1 \mathrm{Mbps}$ bit rate is considered in BPSK. The time period of all signals is taken as $10 \mu$ s with a sampling frequency of $10 \mathrm{MHz}$, $20 \mathrm{MHz}$ and $30 \mathrm{MHz}$ for representation of signal samples $L=100$, 200 and 300 respectively. The SNR is varied from $-10 \mathrm{~dB}$ to $10 \mathrm{~dB}$.

As discussed earlier, the received signal is defined by binary hypothesis as shown in Eq.(5) and Eq.(6)

where $y(n)=$ Received Signal at SU, $W(n)=\mathrm{AWGN}, S_{i}(n)=$ Transmitted Signal by PU (AM or FM or BPSK Modulation).

$$
S_{i}(n)=\left\{\begin{array}{cc}
A M \text { band signal } & \text { if } i=1 \\
F M \text { band signal } & \text { if } i=2 \\
G S M 900 \text { band signal } & \text { if } i=3
\end{array}\right.
$$

The different received can be represented as

$$
\begin{gathered}
S_{1}(n)=A_{c} *\left(1+m^{*} \sin \left(2 \pi f_{a} t\right)\right) * \sin \left(2 \pi f_{c} t\right) \\
S_{2}(n)=\sin \left(2 \pi f_{c} t+\left(m^{*} \sin \left(2 \pi f_{a} t\right)\right)\right) \\
S_{3}(n)=s_{\text {mod }} * \cos \left(2 \pi f_{c} t\right)
\end{gathered}
$$

where $A_{c}=$ Amplitude of carrier signal $=A_{m} / m, A_{m}=$ Amplitude of modulating signal, $m=$ Modulation Index, $f_{a}=$ Frequency of modulating signal, $f_{c}=$ Frequency of carrier signal, $s_{\text {mod }}=$ Bipolar NRZ signal, +1 for logic 1 and -1 for logic 0

Initially 40 received signals for hypothesis $H_{0}$ and 40 received signals for hypothesis $H_{1}$ are simulated. By using Eq.(18) to Eq.(19) features of $y(n)$ is calculated i.e. we get 160 feature values of PU signal and 160 feature values of AWGN. This data is used for all the ML techniques. The performance of each algorithm is calculated below.

\subsection{ENERGY DETECTION}

The Table. 1 shows the error rate of all signals for different SNR values. It is observed that the error in AM modulation is less as compared to FM and BPSK modulation because, in FM and BPSK modulation, the energy of modulating signal and modulated signal is unchanged whereas the energy in AM modulation changes. The error gets reduced as increase the SNR value as well as a number of signal samples $L$. 
Table.1. Error Rate of different SNR values for ED

\begin{tabular}{|c|c|c|c|c|c|c|c|c|c|}
\hline$L$ & \multicolumn{3}{|c|}{100} & \multicolumn{3}{|c|}{200} & \multicolumn{3}{|c|}{300} \\
\hline \multirow{2}{*}{$S(n)$} & \multicolumn{9}{|c|}{$S N R$ in $\mathrm{dB}$} \\
\hline & 0 & -5 & -10 & 0 & -5 & -10 & 0 & -5 & -10 \\
\hline BPSK & 0.1 & 0.5 & 0.8 & 0.1 & 0.3 & 0.7 & 0.1 & 0.2 & 0.7 \\
\hline FM & 0.1 & 0.5 & 0.8 & 0.1 & 0.3 & 0.7 & 0.1 & 0.2 & 0.7 \\
\hline $\mathrm{AM}$ & 0.1 & 0.4 & 0.7 & 0.1 & 0.2 & 0.6 & 0.1 & 0.1 & 0.6 \\
\hline
\end{tabular}

The system performance is evaluated by considering $P_{e}, P_{d}$, $P_{f a}$ and $P_{\text {miss }}$ parameters. The Fig. 3 shows graph of $P_{e}$ vs. SNR of BPSK signal for $L=100,200$ and 300 in which error rate decreases as SNR increases. The system gives the poor performance at low SNR value.

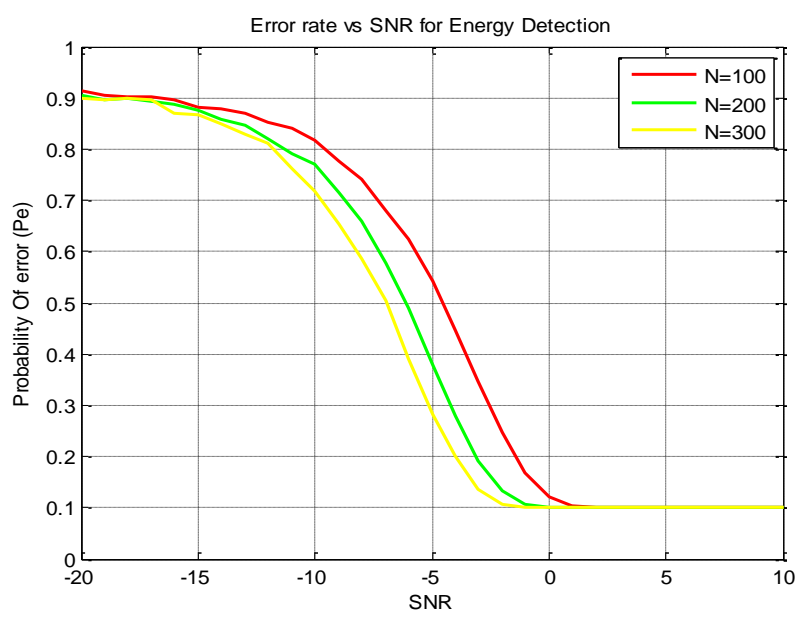

Fig.3. Error Rate vs. SNR of BPSK for ED

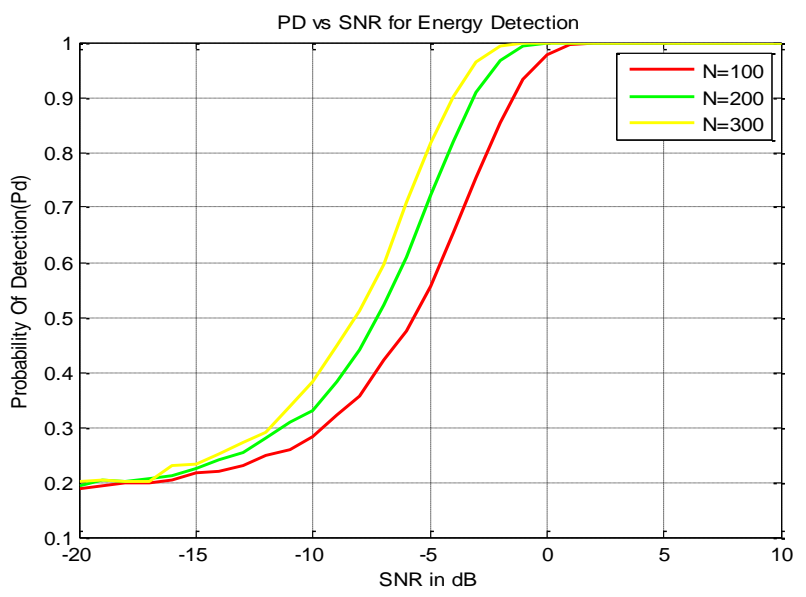

Fig.4. $P_{d}$ vs SNR of BPSK for ED



Fig.5. ROC curve of BPSK for ED

For $S N R=-1 \mathrm{~dB}$ error rate is 0.1 for $L=300,0.1004$ for $L=$ 200 and 0.1500 for $L=100$. The system consists of two types of errors $P_{f a}$ and probability miss detection $\left(P_{m}\right)$ and in this system, the $P_{f a}$ is considered 0.1 , therefore in the system always error will present. The Fig. 3 shows that the minimum error 0.1 i.e. $P_{f a}$ will always present.

As shown in Fig.4, the system gives $P_{d}=1$ at $S N R=-2 \mathrm{~dB}, 0$ $\mathrm{dB}$ and $1 \mathrm{~dB}$ for $N=300,200$ and 100 respectively. The $P_{d}$ shows the probability of correct detection of PU signal so it should be as high as possible.

The Fig.5 shows ROC curve. In Fig.5, the ROC performance of the ED is shown at $S N R=-5 \mathrm{~dB}$ using different $L$. It can be observed that the performance of the scheme is improved as more samples of PU's signals are obtained for the energy calculation. It can be seen that at $P_{f a}$ of $0.1, P_{d}$ rises from about 0.51 to about 0.81 as $L$ is increased from 10 to 300 .

\subsection{ANN}

The error rate of the system is calculated by considering Multiple Features (MF) and Single Feature (SF). In MF, all four features stated in Eq.(18) to Eq.(21) are extracted whereas in SF only energy feature is extracted.

Table.2. Error Rate of different SNR values for ANN (MF)

\begin{tabular}{|c|c|c|c|c|c|c|c|c|c|}
\hline$L$ & \multicolumn{3}{|c|}{100} & \multicolumn{3}{|c|}{200} & \multicolumn{3}{|c|}{300} \\
\hline \multirow{2}{*}{$S(n)$} & \multicolumn{9}{|c|}{$S N R$ in $\mathrm{dB}$} \\
\hline & 0 & -5 & -10 & 0 & -5 & -10 & 0 & -5 & -10 \\
\hline BPSK & 0.0 & 0.1 & 0.4 & 0.0 & 0.0 & 0.3 & 0.0 & 0.0 & 0.1 \\
\hline FM & 0.0 & 0.1 & 0.3 & 0.0 & 0.0 & 0.3 & 0.0 & 0.0 & 0.1 \\
\hline $\mathrm{AM}$ & 0.0 & 0.1 & 0.3 & 0.0 & 0.0 & 0.3 & 0.0 & 0.0 & 0.1 \\
\hline
\end{tabular}


Table.3. Error Rate of different SNR values for ANN (SF)

\begin{tabular}{|c|c|c|c|c|c|c|c|c|c|}
\hline$L$ & \multicolumn{3}{|c|}{100} & \multicolumn{3}{|c|}{200} & \multicolumn{3}{|c|}{300} \\
\hline \multirow{2}{*}{$S(n)$} & \multicolumn{9}{|c|}{$S N R$ in $\mathrm{dB}$} \\
\hline & 0 & -5 & -10 & 0 & -5 & -10 & 0 & -5 & -10 \\
\hline BPSK & 0.0 & 0.1 & 0.4 & 0.0 & 0.0 & 0.4 & 0.0 & 0.0 & 0.1 \\
\hline FM & 0.0 & 0.1 & 0.4 & 0.0 & 0.0 & 0.3 & 0.0 & 0.0 & 0.1 \\
\hline $\mathrm{AM}$ & 0.0 & 0.1 & 0.4 & 0.0 & 0.0 & 0.3 & 0.0 & 0.0 & 0.1 \\
\hline
\end{tabular}

In case of ANN, the received signal's features are extracted and for training one target value is given for all four feature. In simple words target value is defined for all four vectors. So error rate will be less by considering MF as compared to SF. The Table. 2 and Table. 3 shows all the error rate for different conditions. In case of MF error rate is 0 at $S N R=-5 \mathrm{~dB}$ and for $\mathrm{SF}$ error rate is 0 at $S N R=0 \mathrm{~dB}$.

\section{PERFORMANCE CURVE}

The Fig.6 shows the $P_{e}$ vs. SNR of BPSK for ANN, in this 25 hidden layers are considered and each layer contained $(2 *$ training sample +1$)$ i.e. 225 neurons. According to Fig. $6, P_{e}$ is decreased gradually as the increase of SNR. The system gives a good performance from $S N R=-5 \mathrm{~dB}$ at $N=300$. Otherwise, $P_{e}$ becomes 0 at $S N R=-4 \mathrm{~dB}$ and $S N R=-1 \mathrm{~dB}$ for $N=200$ and $N=100$ respectively.

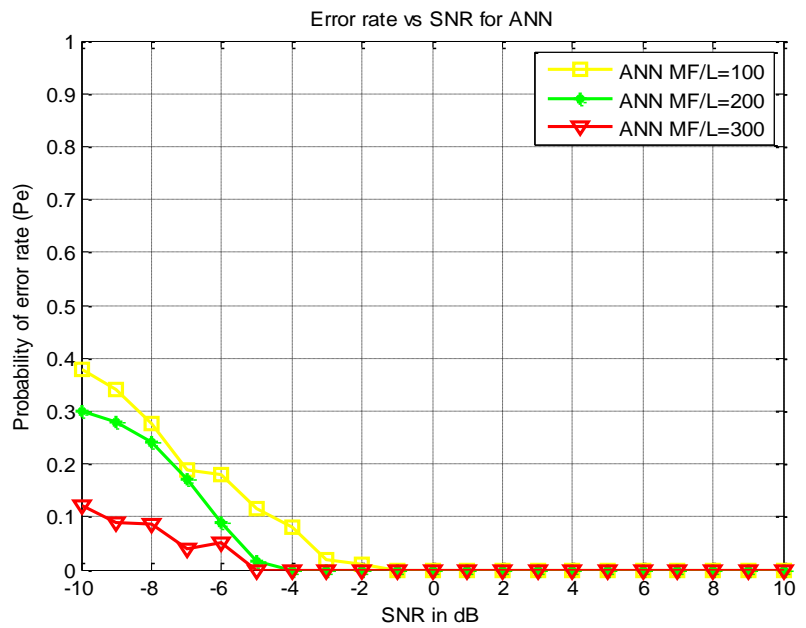

Fig.6. $P_{e}$ vs $S N R$ of BPSK for ANN (MF)

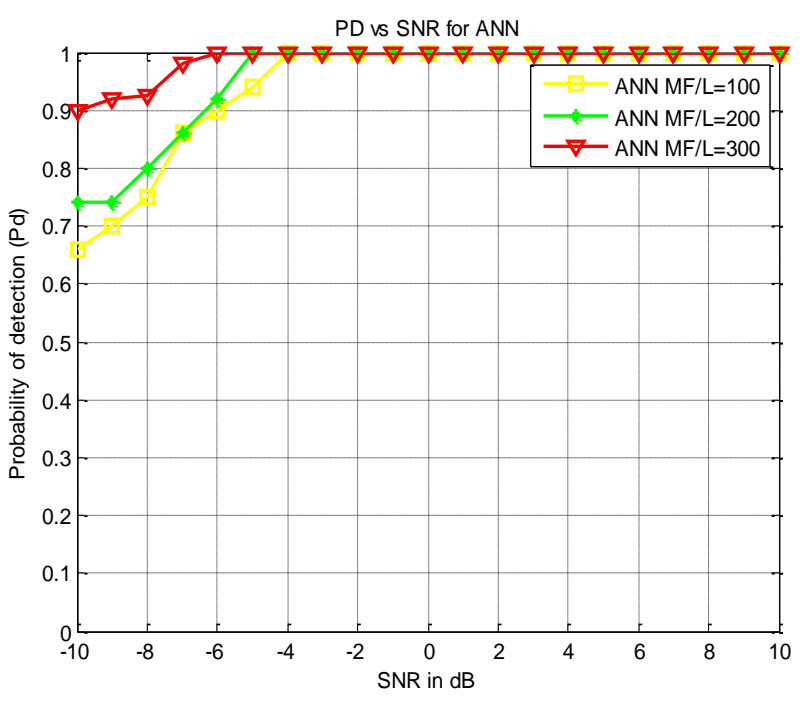

Fig.7. $P_{d}$ vs $S N R$ of BPSK for ANN (MF)

The number of correct and incorrect predictions are summarized with count values and broken down by each class. It shows that the correct detection is $87.5 \%$ and miss classification is 12.5. The same result is shown in Fig.6.

The training process is shown in Fig.9. An epoch is a measure of the number of times all of the training vectors are used once to update the weights. So from Fig.10 system get minimum error at $5^{\text {th }}$ epoch and mean square error is 0.06338 .

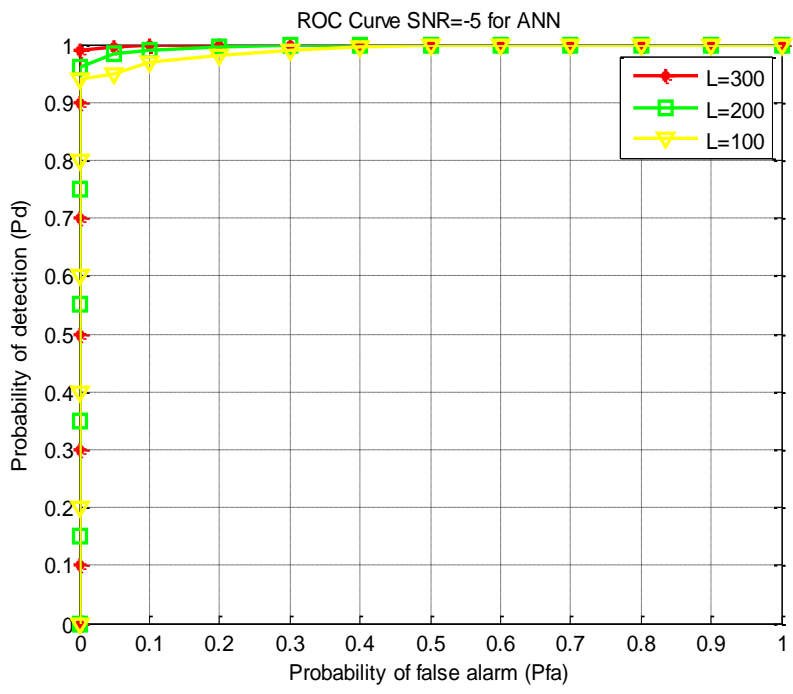

Fig.8. $P_{d}$ vs. $P_{f a}$ of BPSK for ANN (MF) 


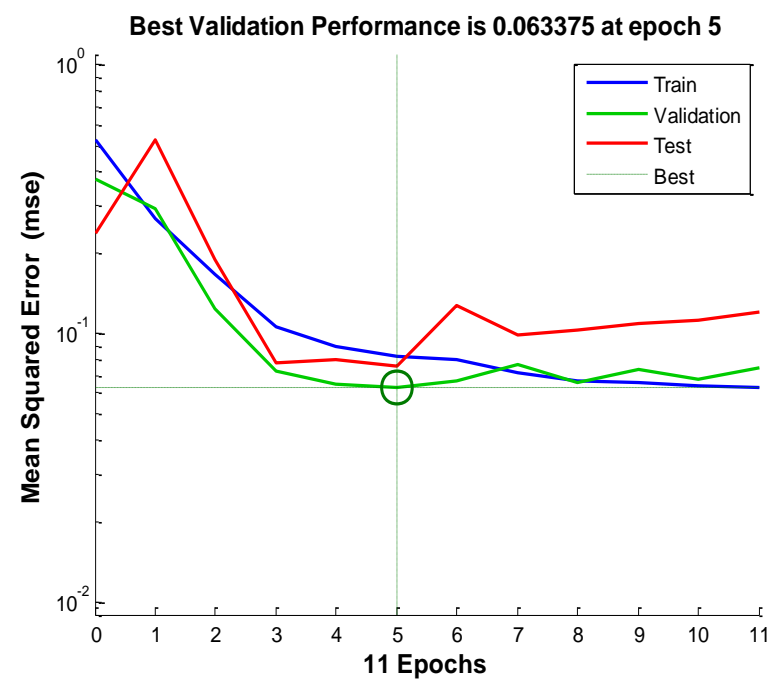

Fig.9. Mean square error for $S N R=-10 \mathrm{~dB}$

\section{CONCLUSION}

In this paper, The ED technique and ANN algorithm is compared for SS. The ANN gives better performance as compared to ED method. The total time require for decision is $1 \mathrm{~s}$ and $14 \mathrm{~ms}$ for ED method and ANN algorithm respectively. The ANN gives good results at low SNR. The zero error rate can achieve at $S N R=-5 \mathrm{~dB}$ when ANN algorithm is used while by using ED method minimum error rate can will be 0.1 . The performance of system depends upon, corresponding threshold value in case of ED method and number of hidden layers in case of ANN algorithm. The $P_{d}=1$ can achieve at $S N R=-1 \mathrm{~dB}$ and $S N R=-6 \mathrm{~dB}$ for ED method and ANN algorithm respectively. At $S N R=-5 \mathrm{~dB}, 100 \%$ accuracy is achieved by using ANN and $70 \%$ accuracy is achieved by using ED. The ED method is less complex as compared to ANN because it does not require training data, priory knowledge of signal and multilayer network.

\section{REFERENCES}

[1] N. Muchandi and R. Khanai, "Cognitive Radio Spectrum Sensing: A Survey", Proceedings of International
Conference on Electrical, Electronics, and Optimization Techniques, pp. 3233-3237, 2016.

[2] M. Ashraf, J. Khan, H. Rasheed, F. Ashraf, M. Faizan and M.I. Anis, "Demonstration of Energy Detector Performance and Spectrum Sensing in Cognitive Radio using AWGN, Rayleigh and Nakagami channels", Proceedings of International Conference on Innovations in Electrical Engineering and Computational Technologies, pp. 1-7, 2017.

[3] M. Bkassiny, Y. Li and S.K. Jayaweera, "A Survey on Machine-Learning Techniques in Cognitive Radios", IEEE Communications Surveys and Tutorials, Vol. 15, No. 3, pp. 1136-1159, 2013.

[4] N. Abbas, Y. Nasser and K.E. Ahmad, "Recent Advances on Artificial Intelligence and Learning Techniques in Cognitive Radio Networks", EURASIP Journal on Wireless Communications and Networking, Vol. 174, pp. 26-46, 2015.

[5] Y.J. Tang, Q.Y. Zhang and W. Lin, "Artificial Neural Network Based Spectrum Sensing Method for Cognitive Radio", Proceedings of $6^{\text {th }}$ International Conference on Wireless Communications Networking and Mobile Computing, pp. 1-4, 2010.

[6] J.J. Popoola and R. Van Olst, "Application of Neural Network for Sensing Primary Radio Signals in a Cognitive Radio Environment", Proceedings of International Workshop on Women in Engineering, pp. 1-6, 2011.

[7] S. Pattanayak and R. Nandi, "Identification of Spectrum Holes using ANN Model for Cognitive Radio Applications", Eurocon 2013, pp. 133-137, 2013.

[8] T. Zhang, M. Wu and C. Liu, "Cooperative Spectrum Sensing Based on Artificial Neural Network for Cognitive Radio Systems", Proceedings of $8^{\text {th }}$ International Conference on Wireless Communications, Networking and Mobile Computing, pp. 1-5, 2012.

[9] R. Singh and S. Kansal, "Artificial Neural Network based Spectrum Recognition in Cognitive Radio", Proceedings of IEEE International Conference on Electrical, Electronics and Computer Science, pp. 1-6, 2016.

[10] V. Gatla, M. Venkatesan and A.V. Kulkarni, "Feed Forward Neural Network based Learning Scheme for Cognitive Radio Systems", Proceedings of IEEE $3^{\text {rd }}$ International Conference on Computational Intelligence and Information Technology, pp. 25-31, 2013. 